\title{
Abus sexuels, un problème à résoudre
}

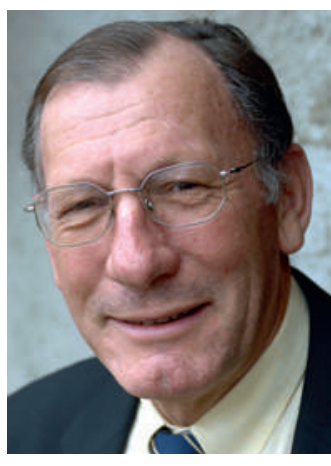

Jean Martin
Les abus de nature sexuelle dans la pratique professionnelle sont une réalité et un vrai problème - il se peut que $10 \%$ d'entre nous cèdent à cette tentation. Il faut féliciter la FMH de l'empoigner et j'ai apprécié l'éditorial sans concession de notre collègue Christine Romann, du Comité central [1].

C'est un de ces sujets que personne n'aime voir: les patient(e)s concerné(e) $s^{*}$ sont traumatisés, inquiets de se voir accusés d'avoir invité l'abus; les médecins ou autres soignants craignent que le débat fasse penser que tous sont coupables; le public est dérangé dans son vœu que les médecins soient techniquement compétents mais aussi irréprochables dans leurs conduites; et les instances de surveillance (sociétés professionnelles et départements de la santé - au premier chef le médecin cantonal) peuvent être réticentes devant des situations de type «la parole de l'un(e) contre la parole de l'autre», qui ne permettent pas de conclure catégoriquement. La supervision de la pratique n'a rien d'aisé, l'essentiel de ce qui se passe entre soigné et soignant l'est dans un cadre très privé. D'où un malaise parce qu'il s'ensuit (statistiquement parlant) que des comportements inacceptables échappent à la nécessaire mise en garde ou sanction.

Christine Romann met le doigt sur un aspect de droit administratif, appliqué aussi par les organes déontologiques, très insatisfaisant alors que la transparence est partout demandée: même quand l'instruction montre que le professionnel a été inadéquat et qu'il fait l'objet de sanction, le/la plaignant(e) n'a jusqu'ici pas connaissance des conclusions de l'autorité mais doit se contenter d'apprendre qu'on a donné suite à son intervention, sans autres détails... C'est ce que la FMH a voulu changer à combien juste titre, en donnant au plaignant le statut de partie. On ne doit pas ici sous-estimer la valeur de réparation (partielle), pour le patient, de connaître la décision. De plus et sans vouloir noircir le tableau, un volet pénal doit être envisagé dans les cas graves; cas où l'administration sanitaire doit en principe transmettre sa décision à l'Ordre judiciaire, mais le plaignant devrait aussi savoir qu'une suite pénale peut se justifier.

Autre point: quand des professionnels critiquables savent que d'éventuelles sanctions sont connues des patients, voire rendues publiques, il y a là un potentiel préventif; on craindra plus de se laisser tenter par des gestes ou paroles discutables. Dans la pratique vaudoise depuis plus de dix ans, les sanctions graves sont publiées dans la Feuille des avis officiels.

$\mathrm{Au}$ plan des codes de déontologie, le Québec a été un des premiers, il y a une vingtaine d'années, à

établir des dispositions fermes. Certaines sont devenues de routine; ainsi la notion qu'un examen vaginal est fait en présence d'une autre personne autorisée, comme une infirmière. A noter aussi le cas (non critiquable en soi) où patient(e) et soignant(e) développent des sentiments du registre amoureux et sexuel: la règle est que le professionnel met un terme au rapport de soins, que durant une période de trois voire six mois les amoureux s'astreignent à ne plus avoir de contacts et qu'après cet intervalle ils décident de leur avenir personnel.

Agir efficacement n'est pas simple: les sociétés et commissions professionnelles peuvent être surchargées; leurs moyens d'instruire les plaintes sont limités - comme le sont ceux des départements cantonaux de la santé qui n'ont pas les compétences d'une police judiciaire. Après s'être plaints, les patients renoncent parfois en découvrant la complexité ou les délais de la procédure. Une autre réalité, je le dis comme je l'ai expérimentée, est qu'un médecin très critiquable peut avoir un très (trop) bon avocat! (et que ce n'est pas forcément vrai du patient).

S'agissant des rôles respectifs des instances professionnelles et de l'Etat, il faut relever que les deux sont nécessaires, ne sont pas interchangeables et ont leur potentiel d'action spécifique. Par exemple: l'Etat ne peut en aucun cas donner une impression d'arbitraire; si une instance administrative, en l'absence de faits bien documentés, se base sur une intime conviction pour punir, elle prend un gros risque de voir un recours aboutir. Mais, une corporation privée peut à mon sens se fonder sur une telle conviction, dans un contexte par essence collégial. NB: il n'y a pas de communication automatique entre société médicale cantonale et département de la santé; cependant, pour le moins dans les cas sérieux, des modalités de transmission doivent être possibles.

Un écueil enfin: si on entend être plus efficace dans la détection d'actes inacceptables, il faut se souvenir qu'on courra le risque de «faux positifs», où la conclusion sera que le médecin n'a rien à se reprocher. Situation qui indignera le praticien, qui aura droit à des excuses. Mais ce sera un prix à payer pour diminuer l'importance du problème.

Jean Martin, ancien médecin cantonal vaudois et membre de la rédaction

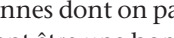
une femme.

Romann C. Abus à caractère sexuel dans l'activité médicale - il faut agir! Bull Méd Suisses. 2012;93(19):703. 